\title{
Reversible Association of Porphyrin Derivative Incorporated into Hydrophobic Region of Phospholipid Bilayer
}

\author{
Lin WANG, Hiroyuki OHNO, ${ }^{\dagger}$ and Eishun TSUCHIDA* \\ Department of Polymer Chemistry, Waseda University, \\ Tokyo 169, Japan
}

(Received December 26, 1988)

\begin{abstract}
Tetramethyl-7,12-divinyl-2,18-bis-(18-hydroxyoctadecyloxycarbonylethyl)porphinato zinc (BHPZn) was incorporated into the hydrophobic bilayer membrane region of liposome of dipalmitoyl phosphatidylcholine (DPPC). Thionine was added to the outer aqueous phase of the liposome suspension. Fluorescence spectra were measured to check the influence of phase of lipid bilayer on the energy transfer from BHPZn to thionine. At temperatures below the phase transition temperature $\left(T_{\mathrm{c}}\right)$ of DPPC liposome, BHPZn was assembled in the bilayer membrane and the excitation energy of BHPZn was dissipated by the static self-concentration quenching. The energy transfer efficiency to thionine was considerably low. Against this, at temperatures above the Tc, concentrated BHPZn was dispersed uniformly and reduced the selfquenching of BHPZn in the excited state. The energy transfer efficiency from porphyrin to thionine increased with heating depending on the fluidity of the lipid bilayer. The fluorescence characteristics of BHPZn reflected the phase behavior of the lipid bilayer, and was simply controlled by changing the physicochemical feature of lipids such as fluidity.

KEY WORDS Porphyrin / Lipid Bilayer / Energy Transfer / Thionine / Phase Behavior
\end{abstract}

By incorporating certain pigments like chlorophylls, porphyrin derivatives, and so on, into the hydrophobic region of liposome bilayer, it is possible to mimic such functions as efficient energy transfer and electron transfer processes for photosyntheses. ${ }^{1-3}$ Energy and electron transfer across the liposome bilayer, on the other hand, can also provide useful information about membrane fluidity and the functions of bilayers. ${ }^{4-8}$ One of the most important characteristics of a liposome bilayer is the gel-to-liquid crystalline phase transition, and non-linear behavior of the incorporated materials is found to be based on the phase change of the matrix. There is much evidence for the localization of some organic pigments, for examples, chlorophylls or 1,6-diphenyl- 1,3,5-hexatriene (DPH) derivatives, in the bilayer membranes, and dye-enriched domains are formed more easily in the rigid gel state of lipid bilayer than in the fluid liquid crystalline phase..$^{9-12}$ In the fluid liquid crystalline state, the molecular motion of both lipid molecules and incorporated compounds is high. Obviously, the interaction between the liposome matrix and incorporated compounds at temperatures below the $T_{\mathrm{c}}$ is different from that at temperatures above the $T_{\mathrm{c}}$ of liposome bilayer. It is essentially important to analyze this phase transition behavior of liposomes for the functional design of metal complexes in the bilayer.

\footnotetext{
${ }^{\dagger}$ Present address: Department of Polymer Engineering, Tokyo University of Agriculture and Technology.

* To whom all correspondence should be addressed.
} 


\section{EXPERIMENTAL}

\section{Materials}

Dipalmitoyl phosphatidylcholine (DPPC) was purchased from Sigma. Thionine was purchased from Kanto Chem. Co., Ltd. 3,8,13,17Tetramethyl-7,12-divinyl-2,18-bis(18-hydroxyloctadecyloxycarbonylethyl)porphinato zinc (BHPZn) was synthesized from protoporphyrin-IX sodium salt according to the method reported elsewhere. ${ }^{12}$

\section{Sample Preparation}

Known amounts of BHPZn and DPPC were dissolved in chloroform. The solution was slowly evaporated with a rotary evaporator to form a transparent thin film on the inner wall of the flask. Distilled water was added and the solution was sonicated (Tomy Seiko UR-200P) for $20 \mathrm{~min}$ at $60 \mathrm{~W}$ in an ice bath under nitrogen atmosphere. The concentration of DPPC was $1.2 \times 10^{-4} \mathrm{M}$ and the ratio of DPPC to BHPZn was $50: 1$.

The incorporation of BHPZn into liposome was first confirmed by GPC and ultracentrifugation. GPC elution curve for BHPZn overlapped completely with that for DPPC liposome. The supernatant of ultracentrifugated liposome suspension contained neither BHPZn nor lipid liposome. Further, spectroscopic study on the polarity around BHPZn revealed that it was incorporated into hydrophobic region. These suggest that BHPZn was entrapped in the liposome matrix. ${ }^{11}$ Transmission electron microscopy (TEM) showed that BHPZn-containing liposome was SUV with an average diameter of about $50 \mathrm{~nm}$.

\section{Measurements}

The BHPZn-containing liposome suspension was incubated overnight at temperature below the phase transition temperature $\left(T_{\mathrm{c}}\right)$ of DPPC liposome before the measurement. Fluorescence spectra were recorded on a JASCO FP-770 Spectrofluorometer with a temperature-controlled unit. The excitation was made at the Soret band of BHPZn of $415 \mathrm{~nm}$. Thionine, an organic pigment, was dissolved in distilled water and added to the liposome suspension as $5.0 \times 10^{-6} \mathrm{M}$. Fluorescence depolarization measurement was made using a polarization filter (JASCO FP2010) after the excitation and before the emission monochrometer. The degree of polarization, $P$, was determined by measurement of the fluorescence intensity with polarization filters placed in all combinations of the horizontal $(h)$ and vertical $(v)$ orientation as seen in eq 1.

$$
\left.P=\left[I_{\mathrm{vv}}-\left(I_{\mathrm{hv}} / I_{\mathrm{hh}}\right) I_{\mathrm{vh}}\right] / / \mathrm{I}_{\mathrm{vv}}+\left(I_{\mathrm{hv}} / I_{\mathrm{hh}}\right) I_{\mathrm{vh}}\right]
$$

\section{RESULTS AND DISCUSSION}

\section{Concentration-dependence of the quenching}

BHPZn bears two long octadecyl chains with hydroxy terminal groups. The lengths of the substituents of this compound are almost comparable to those of a lipid molecule such as DPPC. This feature enables BHPZn to be easily incorporated into the bilayer membrane of liposomes. The DPPC bilayer serves as the matrix to disperse BHPZn in the hydrophobic environment with surrounding highly ordered DPPC molecules. Porphyrin rings of BHPZn tended to orient in the bilayer membrane of liposome, and the terminal hydroxy groups of the two long aliphatic chains faced the aqueous phase. ${ }^{11}$ Incorporation of porphyrins into the lipid bilayer involves binding and partition process. ${ }^{13}$ The distribution of BHPZn in DPPC bilayer is sensitive to the relative concentrations of the solute and lipid under constant temperature. The UV/Vis spectra of BHPZn in DPPC liposomes with different DPPC : BHPZn ratios are shown in Figure 1. A shoulder at about $390 \mathrm{~nm}$ beside the Soret band appeared with decreasing DPPC : BHPZn ratio. This new absorption at $390 \mathrm{~nm}$ was also observed in a micelle and in acetone/ water mixed solvent, and was sensitive to the concentration of BHPZn as well as to the 


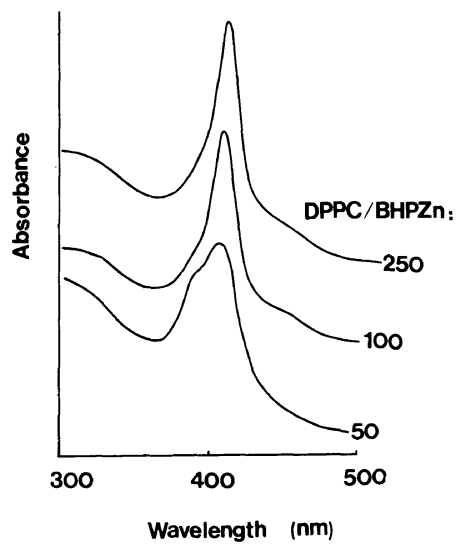

Figure 1. Absorption spectra of BHPZn in DPPC liposomes under different composition at $25^{\circ} \mathrm{C}$. Concentration of BHPZn was set to be $2.0 \times 10^{-6} \mathrm{M}$.

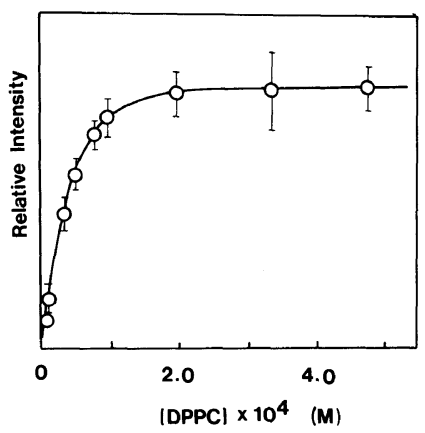

Figure 2. Relationship between fluorescence intensity of BHPZn $\left(\lambda_{\max }=585 \mathrm{~nm}\right)$ and concentration of DPPC. Excited at $415 \mathrm{~nm}, 25^{\circ} \mathrm{C}$.

mixing ratio of the components. Keeping the concentration of $\mathrm{BHPZn}$ constant, an isosbestic point in the UV/Vis spectra was observed at $395 \mathrm{~nm}$ with changing concentration of surfactant or acetone. Absorption is unambiguously attributed to the aggregated porphyrin. ${ }^{14}$ BHPZn tends to aggregate or form porphyrin-enriched local domains in DPPC bilayers when the ratio of DPPC to porphyrin is below $100: 1$ at a temperature below the phase transition temperature $\left(T_{\mathrm{c}}\right)$ of DPPC liposome. ${ }^{11,12}$

Figure 2 shows the relationship between BHPZn fluorescence intensity and concentration of DPPC. The fluorescence spectra

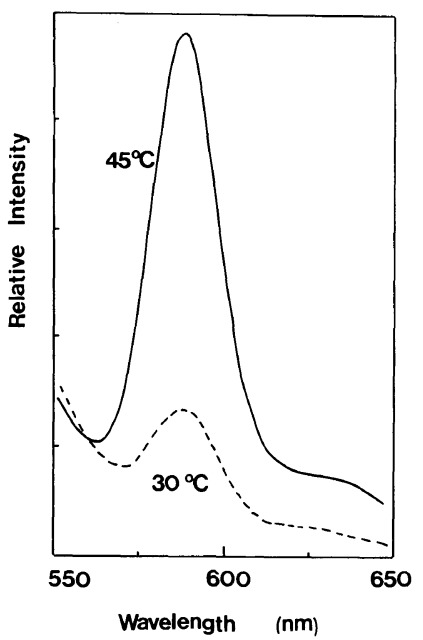

Figure 3. Fluorescence spectra of BHPZn in DPPC liposome at $30^{\circ} \mathrm{C}$ and $45^{\circ} \mathrm{C}$, below and above the $T_{\mathrm{c}}$ of DPPC liposome, respectively. Concentration of BHPZn was $2.0 \times 10^{-6} \mathrm{M}$. Ratio of DPPC: BHPZn was $50: 1$. BHPZn was Excited at $415 \mathrm{~nm}$.

were measured for a series of DPPC liposomes having the same concentration of BHPZn $\left(2.0 \times 10^{-6} \mathrm{M}\right)$ at $25^{\circ} \mathrm{C}$, below the $T_{\mathrm{c}}$ of DPPC liposome. When DPPC: BHPZn ratio was below $100: 1$, fluorescence intensity of BHPZn was quenched. These were attributed to the self-quenching effect or the formation of nonfluorescent aggregates. The fluorescence behavior of BHPZn in DPPC liposome also suggests that there are local BHPZn-enriched domains in the lipid bilayer under these conditions.

\section{Temperature-Dependence of the Quenching}

In addition to the concentration effect, temperature is recognized to be another dominant factor which influences the dispersion state of BHPZn in DPPC bilayer. When the temperature was below the $T_{\mathrm{c}}$ of DPPC bilayer $\left(T_{\mathrm{c}}=\right.$ $41^{\circ} \mathrm{C}$ for LUV and $39^{\circ} \mathrm{C}$ for SUV), the matrix was in a rigid state and the molecular motion of DPPC molecules was considerably suppressed. The fluorescence spectra of BHPZn in a DPPC liposome below and above the $T_{\mathrm{c}}$ of the DPPC liposome are shown in Figure 3. The fluorescence intensity at $45^{\circ} \mathrm{C}$ was about 


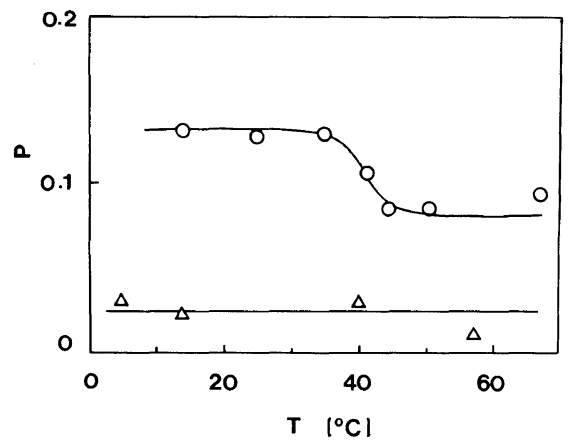

Figure 4. Temperature-dependence of fluorescence polarization $(P)$ of BHPZn in DPPC liposome. BHPZn was incorporated into a DPPC liposome (DPPC: BHPZn ratio $=400: 1)(\bigcirc)$, or $\mathrm{BHPZn}$ was dissolved in ethanol $(\triangle)$.

3 times stronger than that at $30^{\circ} \mathrm{C}$. This suggests that the locally concentrated BHPZn are dispersed at temperature above the $T_{\mathrm{c}}$. The change in the phase state of the matrix perturbed and had impact on the neighboring embedded BHPZn molecules, inducing recovery of suppressed molecular motion of the embedded BHPZn at the rigid gel state of the matrix. As a result, local porphyrin-enriched domains disappeared and the fluorescence intensity obviously increased.

To investigate the interactions between DPPC matrix and BHPZn molecules at temperature below and above $T_{\mathrm{c}}$ of DPPC liposome, fluorescence polarization $(P)$ of $\mathrm{BHPZn}$ in DPPC bilayer was plotted against temperature (Figure 4). The absorption and fluorescence spectra showed that BHPZn was monomerically dispersed in the bilayer membrane of liposome and we can exclude the influence of the concentration as well as temperaturedependent aggregation on the $P$ value. Above the $T_{\mathrm{c}}$, the $P$ value decreased and reached a constant value of about 0.10 . This suggests that the porphyrin ring is indeed incorporated in the matrix of the DPPC bilayer. The environment of BHPZn at temperatures above and below the $T_{\mathrm{c}}$ was revealed to be different, suggesting the stable incorporation of BHPZn into the hydrophobic region of the DPPC

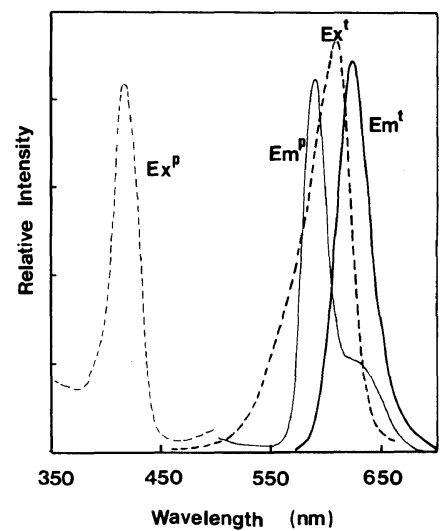

Figure 5. Excitation and emission spectra of BHPZn and thionine. BHPZn and thionine were dispersed in a DPPC liposome and in an aqeuous medium, respectively. Concentration of thionine was $5.0 \times 10^{-6} \mathrm{M}$.

liposome matrix.

\section{Influence of the Phase Transition on Energy Transfer}

The energy transfer efficiency through dipole-dipole interaction decreases by collision between the energy donor and energy acceptor, and the formation of an exciplex. By incorporation of either the donor or acceptor into the bilayer membrane of liposome, it is possible to prevent the concerned molecules from collison and formation of an exciplex. In order to realize such a system, thionine was added to the outer aqueous phase of BHPZncontaining DPPC liposome suspension (DPPC/BHPZn $=50 / 1$ ). The energy transfer from porphyrin to thionine was analyzed. The emission spectrum of BHPZn in DPPC bilayer overlaps with the excitation spectrum of thionine in an aqueous solution (see Figure 5). The porphyrin ring of BHPZn, as the energy donor, was in the bilayer membrane at a certain depth to the interface of the membrane/ aqueous phase, and thionine, as the energy acceptor, was dissolved in the outer aqueous phase. Because BHPZn and thionine are in different phases in this system, the energy transfer through short-distance collision mechanism from BHPZn to thionine is impos- 


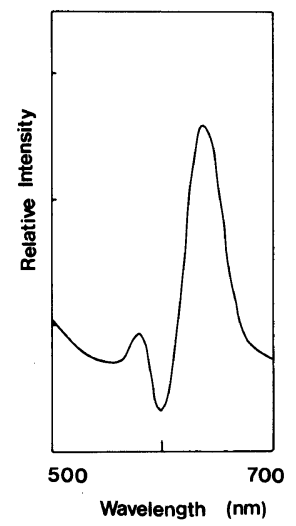

Figure 6. Differential emission spectrum of thionine in BHPZn-containing DPPC liposome suspension. Excitation was made at the Soret band of BHPZn at $415 \mathrm{~nm}$. The differential spectrum was obtained by subtracting the contribution of BHPZn emission.

sible under this condition. The energy of the excited state of BHPZn can only be transferred to thionine via the simple radiative transfer and donor acceptor long range excitation transfer. ${ }^{15}$ When the suspension was excited at the Soret band of BHPZn $(415 \mathrm{~nm})$, an emission attributed to thionine at $631 \mathrm{~nm}$ was observed. Figure 6 shows the emission spectrum of thionine in BHPZn-containing DPPC liposome suspension excited at $415 \mathrm{~nm}$. The spectrum was a differential spectrum obtained by subtracting the influence of BHPZn emission. Thionine shows no observable absorption at $415 \mathrm{~nm}$ and there is no detectable emission from thionine excited by the light with wavelength of $415 \mathrm{~nm}$ in the absence of BHPZn. The perturbation of BHPZn emission tail at $631 \mathrm{~nm}$ on the emission spectrum of thionine was subtracted with BHPZn/DPPC liposome as a reference. The emission of thionine with an excitation at $415 \mathrm{~nm}$ as shown in Figure 6 suggests that the energy transfer took place from BHPZn to thionine. When BHPZn was excited with monochromatic light at $415 \mathrm{~nm}$, the excited BHPZn transferred the energy to thionine via both radiation transfer and no-radiation long range donor-acceptor transfer. Then, the excited thionine dissipated

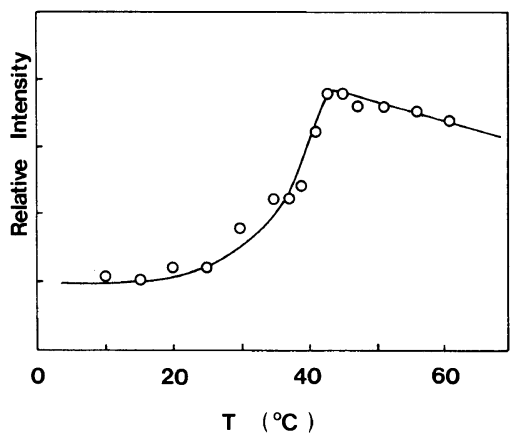

Figure 7. Influence of the temperature of a DPPC suspension on the emission intensity of thionine. Thionine was added to the outer aqeuous phase of BHPZn-containing DPPC liposome suspension. Excitation was made at the Soret band of BHPZn of $415 \mathrm{~nm}$.

the energy of excited state again through the fluorescence emission with $\lambda_{\max }$ at $631 \mathrm{~nm}$.

Figure 7 shows the effect of temperature on the emission intensity at $631 \mathrm{~nm}$ from thionine. The emission intensity increased slowly with increasing temperature, and then there was a sharp increase near the $T_{\mathrm{c}}$ of DPPC. Above the $T_{\mathrm{c}}$ of DPPC liposome, the emission intensity showed a negative slope against temperature according to temperature-enhanced decay process of the excited state. Because thionine was dispersed monomerically under these conditions, the emission intersity from thionine should decrease monotonously with increasing temperature when there is no phase change in the matrix. At the gel state of temperature below $T_{\mathrm{c}}$, the energy of excited BHPZn was dissipated via the non-radiation pathway due to the concentration quenching effect. Thus, there was low efficiency of energy transfer to thionine in the outer aqueous phase via radiation transfer and long-range transfer of Förster mechanism. The localized BHPZn was dispersed gradually by heating and the energy transfer efficiency from BHPZn to thionine also increased correspondingly by the decrease of self-quenching of BHPZn excited state. There was a sharp increase in energy transfer efficiency at temperature near the phase tran- 


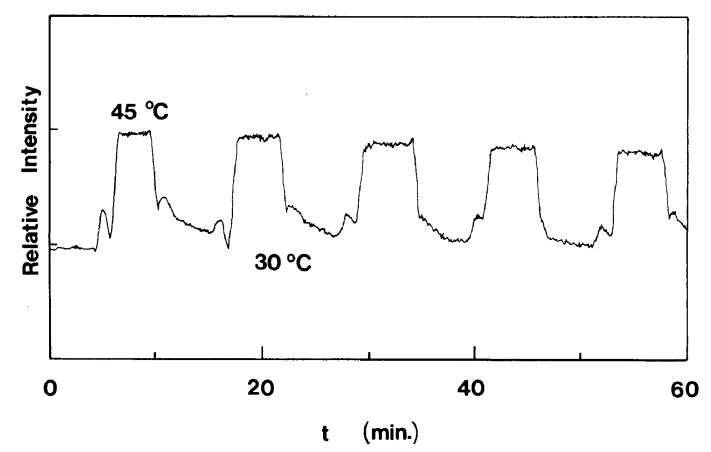

Figure 8. Response of the emission intensity of thionine to temperature. Thionine was dissolved in the outer aqeuous phase of DPPC liposome suspension as $5.0 \times 10^{-6} \mathrm{M}$.

sition region as a result of the drastic phase transition of the matrix.

Phase transition of lipid liposomes is thermodynamically reversible, and the dispersion state of BHPZn in lipid bilayer also reflects this reversible transition. ${ }^{6}$ Figure 8 shows the response of the emission intensity of thionine with excitation at $415 \mathrm{~nm}$ to the temperature change. The emission intensity decreased and reached a certain value by cooling from $45^{\circ} \mathrm{C}$ to $30^{\circ} \mathrm{C}$, below the $T_{\mathrm{c}}$ of DPPC liposome, and then. increased to a higher constant when heated to $45^{\circ} \mathrm{C}$, above the $T_{\mathrm{c}}$ of the matrix. The change in the emission intensity of thionine by the temprature scan is reversible. The energy transfer from BHPZn to thionine thus appears to be strictly regulated by changing the phase state of the matrix.
Acknowledgment. This work was partially supported by a Grant-in-Aid for Scientific Research on Priority Area of "Macromolecular Complexes" No. 62612005 from the Ministry of Education, Science and Culture, Japan.

\section{REFERENCES}

1. M. P. Pileni, Chem. Phys. Lett., 71, 317 (1980).

2. Y. Aso, K. Kano, and T. Matsuo, Biochim. Biophys. Acta, 599, 403 (1980).

3. E. Tsuchida, M. Kaneko, H. Nishide, and $\mathbf{M}$. Hoshino, J. Phys. Chem., 90, 2283 (1986).

4. T. Nomura, J. R. Escabi-Perez, J. Sunamoto, and J. H. Fendler, J. Am. Chem. Soc., 102, 1484 (1980).

5. J. K. Hurley, F. Castelli, and G. Tollin, Photochem. Photobiol., 32, 79 (1980).

6. L. Wang, E. Shouji, H. Ohno, and E. Tsuchida, Polym. Bull. (1989), in press.

7. B. K. Fung and L. Stryer, Biochemistry, 17, 5241 (1978).

8. K. Kano, T. Yamaguchi, and T. Matsuo, J. Phys. Chem., 84, 72 (1980).

9. M. T. Allen, L. Miola, and D. G. Whitten, J. Am. Chem. Soc., 110, 3198 (1988).

10. H. Takemoto, S. Inoue, T. Yasanaga, M. Sukigara, and Y. Toyoshima, J. Phys. Chem., 85, 1032 (1981).

11. L. Wang, T. Takahashi, H. Ohno, and E. Tsuchida, J. Macromol. Sci., Chem. Ed., A26, 481 (1989).

12. L. Wang, H. Ohno, and E. Tsuchida, Bull. Sci. Eng. Res. Lab., Waseda Univ., 121, 8 (1988).

13. M. Rotenberg and R. Margalit, Biochim. Biophys. Acta, 905, 172 (1987).

14. K. A. Zachariasse and D. G. Whitten, Chem. Phys. Lett., 22, 527 (1973).

15. T. Förster, Discus. Faraday Soc., 27, 7 (1959). 